\title{
The effects of spatially correlated heterogeneities on acidising wormhole development
}

\section{Masoud Babaei PhD}

Lecturer in Petroleum Engineering, School of Chemical Engineering and Analytical Science, The University of Manchester, Manchester, UK

(corresponding author: masoud.babaei@manchester.ac.uk) (Orcid:0000-0002-4201-3489)
Majid Sedighi BSc, MSc, PhD

Senior Lecturer in Geotechnical Engineering, School of Mechanical, Aerospace and Civil Engineering, The University of Manchester, Manchester, UK (Orcid:0000-0002-5104-5781)

A commonly used rock stimulation technique in subsurface geoenergy technologies is matrix acidisation. During this process, an acidic solution is injected into the hydrocarbon or geothermal reservoirs to dissolve certain minerals and enhance the injectivity and productivity of the hydrocarbon or heat recovery operation. This study aims to investigate the influence of three-dimensional (3D) lithological heterogeneity of the rock on reactive transport of acid in the porous domain. Through a development and application of a reactive transport model with restricting assumptions (linear calcite dissolution kinetics with hydrochloric acid $(\mathrm{HCl})$ ) and simplifications (mineralogical homogeneity) on the geochemistry embedded into the model, we present the results of a series of 3D simulation of carbonate acidisation in presence of varying spatial correlation lengths of petrophysical properties of the domain. The results are compared with the case where petrophysical properties are distributed randomly throughout the domain. The study provides new insights into the impact of increasing correlation lengths that lack in the existing literature. Under the conditions of case studies, the pore volume of the acid injected reduces 10.5 and $12.2 \%$ for Damköhler number of 100 , when correlation lengths of only 0.0667 and $0.1667 \mathrm{~cm}$ are considered in core of length $5 \mathrm{~cm}$.

\author{
Notation \\ $A_{\mathrm{v}} \quad$ dimensionless interfacial area per unit volume available \\ for a reaction \\ $a_{0} \quad$ initial interfacial area per unit volume available for a \\ surface reaction \\ $a_{\mathrm{v}} \quad$ interfacial area per unit volume available for a surface \\ reaction \\ $C_{0} \quad$ inlet concentration of the acid \\ $C_{\mathrm{f}} \quad$ acid concentration in bulk fluid \\ $C_{\mathrm{s}} \quad$ acid concentration at the solid-fluid interface \\ $C_{\phi_{0} \phi_{0}}$ anisotropic covariance matrix \\ $c_{\mathrm{f}} \quad$ dimensionless acid concentration in bulk fluid \\ $c_{\mathrm{s}}$ dimensionless acid concentration at the solid-fluid \\ interface \\ Da Damköhler number \\ $\boldsymbol{D}_{\mathrm{e}} \quad$ effective dispersion tensor \\ $D_{\mathrm{m}} \quad$ molecular diffusion coefficient \\ $H$ dimensionless effective separation distance \\ $H_{\mathrm{T}}^{2} \quad$ macroscale Thiele modulus \\ $h_{i} \quad$ component in the direction $i$ of the effective separation \\ vector \\ $h_{\mathrm{T}}^{2} \quad$ pore-scale Thiele modulus \\ $\boldsymbol{K} \quad$ permeability tensor \\ $K_{0} \quad$ initial average permeability of rock \\ $k_{\mathrm{c}} \quad$ mass transfer coefficient \\ $k_{\mathrm{s}} \quad$ surface reaction rate constant \\ $L \quad$ length of the core \\ $N_{\text {ac }}$ dimensionless acid capacity number \\ $P \quad$ pressure \\ $P_{\text {out }} \quad$ pressure at the exit boundary of the core
}

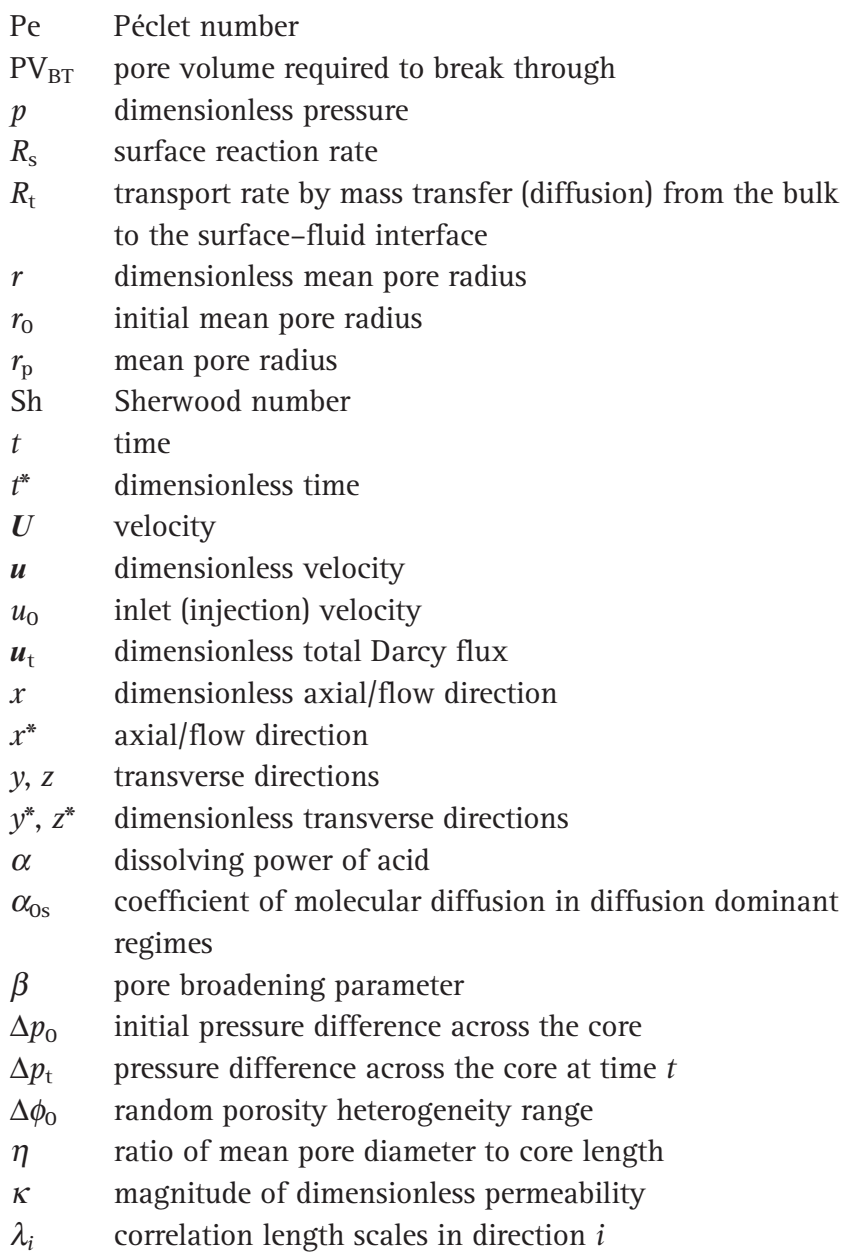


$\mu_{\phi_{0}} \quad$ mean of the correlated $\phi_{0}$ field

$\xi_{\mathrm{T}} \quad$ coefficient of transverse hydraulic dispersion in advection-controlled regimes

$\xi_{\mathrm{X}} \quad$ coefficient of longitudinal hydraulic dispersion in advection-controlled regimes

$\rho_{\mathrm{s}} \quad$ solid phase density

$\sigma_{\phi_{0}}^{2} \quad$ variance of $\phi_{0}$

$\phi \quad$ evolving porosity of the rock

$\phi_{0} \quad$ initial mean porosity

\section{Introduction}

Rock matrix acidisation is a process by which the sediments, mud solids and minerals within the pores of siliciclastic or carbonate reservoirs are dissolved by injecting an acidic solution into the rock below the fracturing pressure level. The rock-matrixacidising process can enhance the injectivity and productivity of fluid and gas recovery operations in applications including hydrocarbon or geothermal enhanced recovery. The process features a strongly coupled flow and geochemistry, where the physical properties of rock such as porosity and permeability dynamically evolve across multiple time and length scales.

Reactions between chemical solutions and the solid phase appear in various engineering and natural subsurface processes, including irrigating water discharge to aquifer systems (Valdes-Abellan et al., 2017), karst formation (Evans and Lizarralde, 2003; Zhao et al., 2013), chemical interaction in saturated and unsaturated soils (Atchley et al., 2014; Chen et al., 2009; Cubillas et al., 2005; Ouhadi et al., 2006), radioactive waste containment and remediation (Sedighi et al., 2015, 2018; Spycher et al., 2003), geologic sequestration of carbon dioxide (e.g. (Islam et al., 2014, 2016; Kampman et al., 2014; Rochelle et al., 2004)), acid injection for enhanced oil recovery (e.g. the paper of Ghommem et al. (2015) and references therein) and acid-based enhanced geothermal system (Na et al., 2016; Portier and Vuataz, 2010; Portier et al., 2007; Xiong et al., 2013).

Over a span of decades, a compelling body of works has focused on developing transport modelling tools, tested/validated against experiments to predict wormhole formation, propagation and stability in the context of oil recovery (Bastami and Pourafshary, 2016; Cohen et al., 2008; Daccord et al., 1989; Fredd and Fogler, 1998; Ghommem et al., 2015; Glasbergen et al., 2009; Golfier et al., 2006; Kalia and Balakotaiah, 2007; Liu and Liu, 2016; Liu et al., 2017; Maheshwari et al., 2013; Nierode et al., 1972; Qiu et al., 2013; Schechter and Gidley, 1969). In petroleum engineering, acid solutions (typically $15 \mathrm{wt} \%$ (Hung et al., 1989)) are used to restore or enhance oil production that is affected by the particles that migrated along the flow and eventually accumulated near the wellbore region during the production lifetime. The preferential dissolution pathways are generated only if certain conditions of rock, acid and injection exist. The parameters and conditions of rock that control efficiency include mineralogical configuration and distribution and petrophysical properties and distributions of rock. The right acid type, volume, dissolving power and injection rate are also controlling properties for efficient wormhole generation. Therefore, the interplay between flow and geochemistry determines the states at which wormholes are generated. The interplay is analysed by describing the parameters of flow and geochemistry through the Péclet number (Pe) and Damköhler number (Da).

Porosity and permeability heterogeneity in the carbonate reservoir play an important role in the formation of wormholes during the reactive dissolution process of rock acidisation. The naturally occurring heterogeneities lead to an uneven permeability increase at the solid-liquid interface. The heterogeneity of reservoir porosity and permeability has been commonly described with a random number in previous works that have dealt with modelling of wormhole formations (see e.g. the papers of Panga et al. (2005), Kalia and Balakotaiah (2009) and Maheshwari and Balakotaiah (2013a)). There are very limited studies that have implemented a realistic distribution of petrophysical properties. The most important difference between a realistic distribution of petrophysical properties and a random distribution is the presence of a spatial correlation with different length scales in the porous system. Liu et al. (2012) adopted a normal distribution within a two-dimensional (2D) radial system with circumferential and radial correlations. A spatial correlation through sequential Gaussian simulation has been used by Liu et al. (2016) to generate the initial porosity distribution. In neither of these works has the influence of varying/increasing correlation lengths in the model been investigated - that is, if a comparison has been made between uniform distribution porosity and permeability, a comparison between varying correlation lengths has not been made. Moreover, the impact of correlation length on the development of wormholes and in particular the optimal pore volume $\left(\mathrm{PV}_{\mathrm{BT}}\right)$ is not clarified by Liu et al. (2016) (see Figure 16 of their paper).

The study presented here aims to address the aforementioned shortcoming in literature - that is, how different values of correlation length impact the wormhole development. The authors specifically look at the simplified reaction of calcite with hydrochloric acid $(\mathrm{HCl})$ for a fully homogeneous calcite system, but with heterogeneous rock properties (porosity/permeability). All the assumptions made throughout this paper were justified previously by Panga et al. (2005). The authors use an in-house simulation code (based on the two-scale model of Panga et al. (2005)). In the following, a critical literature review of the modelling approaches using random or correlated porosity and/or permeability is first described (see the section headed 'Heterogeneities of rock and modelling approaches'). Then, the development of a reactive transport model and the governing equations used in the simulation of rock matrix acidisation in this study and the algorithm for generating correlated random fields are described (see the section headed 'Methodology and the simulation model'). The results are presented and discussed in the section headed 'Results and discussions', followed by the section headed 'Conclusions and future works'. 


\section{Heterogeneities of rock and modelling approaches}

Early-stage simulations of porosity heterogeneity have been performed using the average continuum model for carbonate dissolution which is based on the Darcy scale and the pore scale for a linear flow (Panga et al., 2005). The initial porosity field consists of generation of random values fluctuating between a range around the mean value of porosity, which are added to the average porosity - that is, $\left[\phi_{0}-\Delta \phi_{0}, \phi_{0}+\Delta \phi_{0}\right]$. This method is referred to as uniform distribution method that considers a magnitude of randomly generated heterogeneity $\left(\Delta \phi_{0} / \phi_{0}\right)$ within the domain. Permeability is directly related to porosity using semi-empirical relations (e.g. cubic relationship). Kalia and Balakotaiah (2007) extended this modelling approach for a radial flow and showed that a critical value of randomly generated heterogeneous porosity exists that below which the minimum pore-volume-to-breakthrough $\left(\mathrm{PV}_{\mathrm{BT}}\right)$ is much higher than that of a more heterogeneous porous medium. The critical value indicates the minimum amount of heterogeneity required to create instability in the dissolution front and transition from the planar face dissolution to the formation of wormholes. Kalia and Balakotaiah (2009) conducted an investigation on the effect of medium heterogeneities, such as magnitude and length scale over which the porosity varies, and their effect on reactive dissolution for linear flow in a three-dimensional (3D) domain. They showed that heterogeneity affects the structure of the dissolution patterns and, more importantly from a practical point of view, the amount of acid required to achieve a given increase in permeability. They suggested that an optimum heterogeneity magnitude value at which the least amount of $\mathrm{PV}_{\mathrm{BT}}$ is required may exist. Additionally, they demonstrated that the amount of acid required to break through is dependent on the initial rock porosity and dimensions of rock being acidised.

Cohen et al. (2007) proposed a dual-porosity approach using the average-continuum model and considered the reactive medium to consist of two media with different porosities. One medium contains the dominant growing wormholes, and within the other medium, only the short-lived wormholes (and compact dissolution) can occur. Cohen et al. (2007) presented the first 3D simulations of the radial flow, and their model is able to simulate the dissolution patterns known from experiments. Both Cohen et al. (2008) and Kalia and Balakotaiah (2009) showed that the optimum rate or minimum $\mathrm{PV}_{\mathrm{BT}}$ was not affected by the domain size.

Liu et al. (2012) introduced the heterogeneity of porosity to be distributed normally as opposed to be distributed uniformly. In the 2D average continuum radial flow model presented by Liu et al. (2012), it is shown that when considering normally distributed porosities, less $\mathrm{PV}_{\mathrm{BT}}$ is required compared with when the uniform distribution method is applied. In addition, the optimum injection velocity was found to be lower. Acid flows into high-porosity regions, causing wormholes to develop more unevenly compared to the uniform distribution method. It is shown that a large increase in the circumferential correlation length leads to thicker wormhole geometry and greater diversion of propagation direction of the wormholes. A large increase in the radial correlation length causes formation of an increased number of wormholes and thinner wormholes, which may actually reduce the efficiency of the operation. A critical value for the standard deviation (i.e. range of porosities) was derived by Liu et al. (2012). Above this value, $\mathrm{PV}_{\mathrm{BT}}$ decreases sharply, and below this value, it is insensitive. $\mathrm{PV}_{\mathrm{BT}}$ is least when the standard deviation is unity.

The average continuum modelling approach was extended to 3D simulations by Cohen et al. (2008), Maheshwari and Balakotaiah (2013b) and De Oliveira et al. (2012). The effect of large-scale porosity-permeability heterogeneity has been investigated through assessment of vuggy-type carbonates (where cave-like porous features exist in the porous media) and fractures (Izgec et al., 2010; Kalia and Balakotaiah, 2009; Liu et al., 2012). They showed that when incorporating vugs, the acid propagates through the wormholes at a much faster rate than in homogeneous rocks. Acid follows a preferential flow path, guided by the vug network, leading to a decrease in $\mathrm{PV}_{\mathrm{BT}}$. Their model, however, does not consider the acid-rock reaction mechanism (Izgec et al., 2008). Later, Izgec et al. (2010) demonstrated through using Darcy-Brinkman formulations for flow that $\mathrm{PV}_{\mathrm{BT}}$ is influenced by the connectivity of vuggy pores and its spatial distribution in the domain. Maheshwari and Balakotaiah (2013a) demonstrated that for the 3D simulations, a critical porosity heterogeneity $\left(\Delta \phi_{0}\right)$ exists at which $\mathrm{PV}_{\mathrm{BT}}$ is minimum. An increase in the branching and fractal nature of the wormholes is apparent when the randomly generated heterogeneity magnitude $\left(\Delta \phi_{0}\right)$ is increased (Maheshwari and Balakotaiah, 2013a).

More recently, Liu et al. (2016) investigated the effect of incorporating the correlation spatial distribution of the rock petrophysical properties. Lithologically, the authors considered a uniformly distributed porosity of $0 \cdot 2 \pm \Delta \phi_{0}$ and three cases of correlated porosity fields with average porosities of $0 \cdot 2038$, $0 \cdot 2010$ and $0 \cdot 2029$ and similar unreported correlation lengths. The authors considered two different rock-types (dolomite and limestone), with different solubility but with similar porosity/ permeability and that are located close to each other. Mineral heterogeneity is established through simulation of grid cells with different solid volumes. The initial porosity distribution is spatially correlated through the use of sequential Gaussian simulation. The authors demonstrated that the location of wormhole propagation paths is highly influenced by the locality of spatially correlated high-porosity features. However, as stated in the section headed 'Introduction', the correlation length is kept constant (for all the correlated cases) and, in practice, the comparison is based on one random distribution and three correlated cases with a similar spatial correlation length. Moreover, the conclusion made in the paper that 'that larger pore scale heterogeneity leads to a smaller $\mathrm{PV}_{\mathrm{BT}}$ ' is not well established in results (Liu et al., 2016: p. 91; Figure 16). Therefore, using variably correlated heterogeneities, the authors investigate how different correlation lengths may affect wormhole 
development and $\mathrm{PV}_{\mathrm{BT}}$, in an approach more systematic than that of Liu et al. (2016).

\section{Methodology and the simulation model}

Based on the two-scale average continuum model and a dimensionless form of the governing equations, a two-phase reactive transport model has been developed (Babaei and Sedighi, 2018). The formulation facilitates a comparative setting for obtaining the ideal Pe-Da regime at which the effective wormholing can be achieved (see Figure 1). The governing equations are presented briefly here. Details of the theoretical development and numerical model can be found in the paper of Babaei and Sedighi (2018). In deriving these equations, most importantly the reaction of calcite with HCL solution is simplified into experimentally driven linear kinetics (Alkattan et al., 1998). These simplifications have previously been used successfully for carbonate matrix acidisation modelling validated by experimental results (Ghommem et al., 2015).

I. $2 \mathrm{H}^{+}+\mathrm{CaCO}_{3} \rightleftharpoons \mathrm{Ca}^{2+}+\mathrm{CO}_{2}+\mathrm{H}_{2} \mathrm{O}$

1. $R_{\mathrm{s}}=k_{\mathrm{s}}^{\prime}\left(a_{\mathrm{H}^{+}, \mathrm{s}}\right)^{n}=k_{\mathrm{s}}^{\prime}\left(\gamma_{\mathrm{H}^{+}, \mathrm{s}} C_{\mathrm{H}^{+}, \mathrm{s}}\right)^{n}$

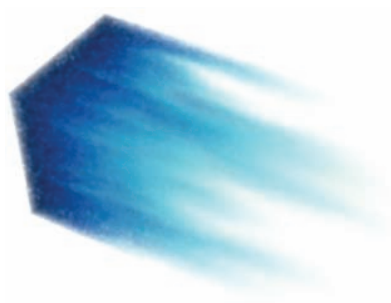

(a)

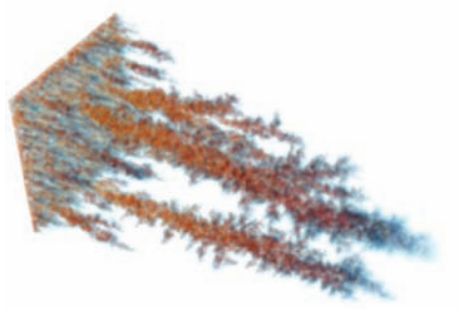

2. $R_{\mathrm{t}}=k_{\mathrm{c}}\left(C_{\mathrm{H}^{+}, \mathrm{b}}-C_{\mathrm{H}^{+}, \mathrm{s}}\right)$

where $R_{\mathrm{S}}$ is the surface reaction rate which is a function of the reaction rate constant, activity of $\mathrm{H}^{+}\left(\gamma_{\mathrm{H}^{+}, \mathrm{s}}\right)$ and surface concentration of $\mathrm{H}^{+}\left(C_{\mathrm{H}^{+}, \mathrm{s}}\right)$, and $R_{\mathrm{t}}$ is the transport rate by mass transfer (diffusion) from bulk to the surface-fluid interface. At the stationary state, the quantity of hydrogen ions transported to the surface equals that consumed by the reaction (Alkattan et al., 1998). Assuming that the exponent of the reaction rate with respect to the concentration of acid is $\sim 1$

3. $R_{\mathrm{t}}=R_{\mathrm{s}}=\frac{k_{\mathrm{c}} k_{\mathrm{s}}^{\prime} \gamma_{\mathrm{H}^{+}, \mathrm{s}}}{k_{\mathrm{s}}^{\prime} \gamma_{\mathrm{H}^{+}, \mathrm{s}}+k_{\mathrm{c}}} C_{\mathrm{H}^{+}, b}=\frac{k_{\mathrm{c}} k_{\mathrm{s}}}{k_{\mathrm{s}}+k_{\mathrm{c}}} C_{\mathrm{f}}$

where $k_{\mathrm{c}} k_{\mathrm{s}} /\left(k_{\mathrm{s}}+k_{\mathrm{c}}\right)$ is the overall (observed) calcite dissolution rate constant that is independent of solution composition, but to a large extent dependent on the experimental design (Alkattan et al., 1998). Therefore, there are uncertainties in this variable that are embedded into the range of Damköhler numbers in this study.

To use the dimensionless equations, a series of dimensionless variables are defined as reported in Table 1. Despite the variations

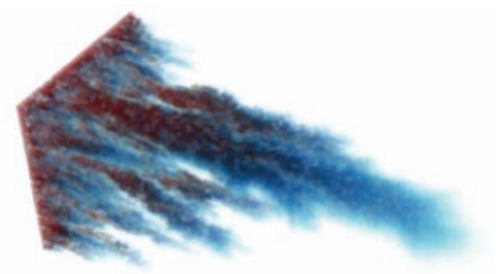

(b)

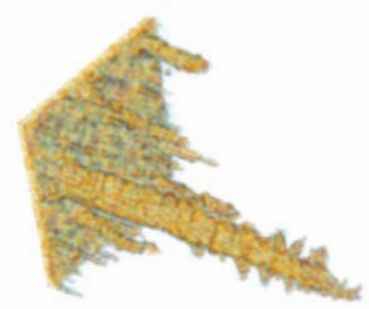

(d)

Absolute porosity increase

Figure 1. Schematic diagram showing the transformation of the dissolution regime from uniform $(\mathrm{Da}=1)$ to wormholing $\left(\mathrm{Da}=10^{4}\right)$ in a 3D modelling example: (a) uniform dissolution $(\mathrm{Da}=1)$; (b) ramified wormholes $(\mathrm{Da}=40)$; (c) wormhole dissolution $(\mathrm{Da}=500)$; (d) conical dissolution $\left(\mathrm{Da}=10^{4}\right)$ (for generating this figure. the code developed in this work has been applied for a single-phase reactive transport problem, the specification of the porous media is the same as that of Panga et al. (2005) extended to three dimensions) 
The effects of spatially correlated

heterogeneities on acidising wormhole

development

Babaei and Sedighi
Table 1. Dimensionless variables

\begin{tabular}{|c|c|}
\hline Variable & Description \\
\hline$x^{*}=x / L, y^{*}=y / L, z^{*}=z / L$ & $\begin{array}{l}\text { Dimensionless position with respect to } \\
\text { the characteristic length } L\end{array}$ \\
\hline$t^{\star}=t /\left(L / u_{0}\right)$ & $\begin{array}{l}\text { Dimensionless time with respect to the } \\
\text { inlet velocity, } u_{0}\end{array}$ \\
\hline $\boldsymbol{u}_{\mathrm{t}}=\boldsymbol{U}_{\mathrm{t}} / \mathrm{u}_{0}$ & Dimensionless total Darcy flux \\
\hline$r=r_{\mathrm{p}} / r_{0}$ & Dimensionless average pore radius \\
\hline$A_{v}=a_{v} / a_{0}$ & Dimensionless interfacial area \\
\hline$\kappa=K / K_{0}$ & Dimensionless permeability \\
\hline$C_{f}=C_{f} / C_{0}, C_{s}=C_{s} / C_{0}$ & $\begin{array}{l}\text { Dimensionless concentrations with } \\
\text { respect to the inlet concentration of } \\
\text { the acid, } C_{0}\end{array}$ \\
\hline$p=\left(P-P_{\text {out }}\right) /\left(u_{0} L / K_{0}\right)$ & $\begin{array}{l}\text { Dimensionless pressure with respect to } \\
\text { the constant pressure at boundary, } \\
P_{\text {out }}\end{array}$ \\
\hline$h_{\mathrm{T}}^{2}=2 k_{\mathrm{s}} r_{0} / D_{\mathrm{m}}$ & $\begin{array}{l}\text { Pore-scale Thiele modulus (reaction } \\
\text { rate over diffusion rate) with respect } \\
\text { to molecular diffusion } D_{m}\end{array}$ \\
\hline$H_{T}^{2}=k_{s} a_{0} L^{2} / D_{m}=$ & Macroscopic Thiele modulus \\
\hline $\mathrm{Da}=k_{\mathrm{s}} \mathrm{a}_{0} L / u_{0}$ & Damköhler number \\
\hline$N_{\mathrm{ac}}=\alpha \mathrm{C}_{\mathrm{o}} / \rho_{\mathrm{s}}$ & Acid capacity number \\
\hline$\eta=2 r_{0} / L$ & $\begin{array}{l}\text { Ratio of average pore diameter to } \\
\text { characteristic length }\end{array}$ \\
\hline$S h=2 k_{c} r_{p} / D_{m}$ & $\begin{array}{l}\text { Sherwood number (the ratio of the } \\
\text { convective mass transfer to the rate } \\
\text { of diffusive mass transport) }\end{array}$ \\
\hline
\end{tabular}

in the mass transfer coefficient, the authors use a constant value $\left(\mathrm{Sh}_{\infty}=3\right)$. Therefore, in the dimensionless analysis, the Sherwood number reflects the value of the mass transfer coefficient. Similarly, the Damköhler number is a reflection of $k_{\mathrm{s}}$.

Based on the two-scale formulation of Panga et al. (2005), the dimensionless flow and transport equations can be simplified to

4. $\boldsymbol{u}_{\mathrm{t}}=-\kappa \nabla^{*} p$

5. $\frac{\partial \phi}{\partial t^{*}}+\nabla^{*} \cdot \boldsymbol{u}_{\mathrm{t}}=0$

$$
\begin{aligned}
& \frac{\partial\left(\phi c_{\mathrm{f}}\right)}{\partial t^{*}}+\frac{\boldsymbol{u}_{\mathrm{t}} \partial\left(c_{\mathrm{f}}\right)}{\partial x^{*}} \\
& =\frac{\partial}{\partial x^{*}}\left[\left(\phi \frac{\mathrm{Da}}{H_{\mathrm{T}}^{2}} \alpha_{0 \mathrm{~s}}+\xi_{\mathrm{X}}\left|\boldsymbol{u}_{\mathrm{t}}\right| \eta r\right) \frac{\partial c_{\mathrm{f}}}{\partial x^{*}}\right] \\
& +\frac{\partial}{\partial y^{*}}\left[\left(\phi \frac{\mathrm{Da}}{H_{\mathrm{T}}^{2}} \alpha_{0 \mathrm{~s}}+\xi_{\mathrm{T}}\left|\boldsymbol{u}_{\mathrm{t}}\right| \eta r\right) \frac{\partial c_{\mathrm{f}}}{\partial y^{*}}\right] \\
& +\frac{\partial}{\partial z^{*}}\left[\left(\phi \frac{\mathrm{Da}}{H_{\mathrm{T}}^{2}} \alpha_{0 \mathrm{~s}}+\xi_{\mathrm{T}}\left|\boldsymbol{u}_{\mathrm{t}}\right| \eta r\right) \frac{\partial c_{\mathrm{f}}}{\partial z^{*}}\right] \\
& -\frac{A_{\mathrm{v}} c_{\mathrm{f}} \mathrm{Da}}{1+h_{\mathrm{T}}^{2} / \mathrm{Sh}}
\end{aligned}
$$

The dimensionless porosity development equation is written as

$$
\text { 7. } \frac{\partial \phi}{\partial t}=\frac{A_{\mathrm{v}} N_{\mathrm{ac}} c_{\mathrm{f}} \mathrm{Da}}{1+h_{\mathrm{T}}^{2} r / \mathrm{Sh}}
$$

The following semi-empirical equations are used between the porosity, permeability, average pore radius and surface area of the rock

8. $\kappa=\frac{\phi}{\phi_{0}}\left[\frac{\phi\left(1-\phi_{0}\right)}{\phi_{0}(1-\phi)}\right]^{2 \beta}$

9. $r=\sqrt{\frac{\kappa \phi_{0}}{\phi}}$

10. $A_{\mathrm{v}}=\frac{\phi}{\phi_{0} r}$

These relationships were used by Panga et al. (2005) and Maheshwari et al. (2013) to model carbonate rocks acidising. If $\beta=1$, the permeability evolution reduces to the wellknown Kozeny-Carman correlation by Wyllie and Gardner (1958): $\kappa \propto \phi^{3} /(1-\phi)^{2}$. Panga et al. (2005) extended the Kozeny-Carman correlation to a dissolving medium by including $\beta$ in the relationships.

The correlated porosity-permeability fields are generated using the field generator developed by Nowak et al. (2008). The authors used fast Fourier transform based on the power spectral estimation method that estimates the spectral density function from a random autocorrelated field. The anisotropic covariance matrix for a second-order stationary field is defined using exponential, Gaussian or spherical methods (Nowak et al., 2008). In this work, the authors use the exponential covariance matrix to produce correlated maps of $\phi_{0}$, given as

11. $C_{\phi_{0} \phi_{0}}(H) \sigma_{\phi_{0}}^{2} \exp (-H)$

where $\sigma_{\phi_{0}}^{2}$ is the variance of $\phi_{0}$ and $H$ is the dimensionless/ effective (anisotropic) separation distance scaled by the correlation length scales $\lambda_{i}[L], i=x, y, z: H=\left(h_{\mathrm{x}}^{2} / \lambda_{\mathrm{x}}^{2}+h_{\mathrm{y}}^{2} / \lambda_{\mathrm{y}}^{2}+\right.$ $\left.h_{\mathrm{z}}^{2} / \lambda_{\mathrm{z}}^{2}\right)^{0.5}$, where $h_{i}[L]$ is the separation vector component in the direction $i$.

The mean of $\phi_{0}, \mu_{\phi_{0}}$, is added to the fast Fourier transform of the random autocorrelated field generated from $C_{\phi_{0} \phi_{0}}$ that has a zero mean.

The variables required for modelling the wormhole development based on the dimensionless equations are assigned values as 
Table 2. Variables and their magnitudes used in the simulation

\begin{tabular}{|c|c|c|}
\hline Variable & Magnitude & $\begin{array}{c}\text { Values by Ghommem } \\
\text { et al. (2015) }\end{array}$ \\
\hline$L_{x}$ & $5 \mathrm{~cm}$ & $30 \mathrm{~cm}$ \\
\hline$\hat{L_{y}}, L_{z}$ & $2 \mathrm{~cm}$ & $3 \cdot 3 \mathrm{~cm}$ \\
\hline$h_{x}, h_{y}, h_{z}$ & $0.0333 \mathrm{~cm}$ & - \\
\hline$r_{0}$ & $0.5 \times 10^{-6} \mathrm{~m}$ & $0.5 \times 10^{-6} \mathrm{~m}$ \\
\hline$\beta$ & 2 & 9 \\
\hline$\alpha_{0 s}$ & 0.4 & 0.5 \\
\hline$\xi_{\mathrm{x}}$ & 0.5 & 0.5 \\
\hline$\xi_{\mathrm{T}}$ & $0 \cdot 1$ & $0 \cdot 1$ \\
\hline Sh & 3 & 3 \\
\hline $\mathrm{Da}$ & 10,100 and 1000 & $0.1<\mathrm{Da}<10^{6}$ \\
\hline$h_{\mathrm{T}}^{2}$ & 0.07 & 0.007 \\
\hline$H_{T}^{2}$ & $10^{6}$ & $2 \cdot 14 \times 10^{4}$ \\
\hline$N_{\mathrm{ac}}$ & $0 \cdot 1$ & 0.05 \\
\hline
\end{tabular}

reported in Table 2. The values are compared with experimental data from core experiments of Ghommem et al. (2015) for $15 \% \mathrm{HCL}$ solution injected into carbonate rock at $65^{\circ} \mathrm{C}$ and with $1 \mathrm{ml} / \mathrm{min}<u_{0}<25 \mathrm{ml} / \mathrm{min}$.

The HCL with dissolving power of $N_{\mathrm{ac}}$ is injected into a purely homogeneous calcite mineral which its dissolution by acid is governed by Da everywhere. The three Da values of 10, 100 and 1000 are used to represent change in the reaction rate constant $\left(k_{\mathrm{s}}\right)$ or initial interfacial area per unit volume available for surface reaction $\left(a_{0}\right)$. This range of values for $\mathrm{Da}$ agrees with the experimental data provided by Ghommem et al. (2015) (see Figure 5 of their paper). There, the authors change $u_{0}$ to have $0 \cdot 1<\mathrm{Da}<10^{6}$.

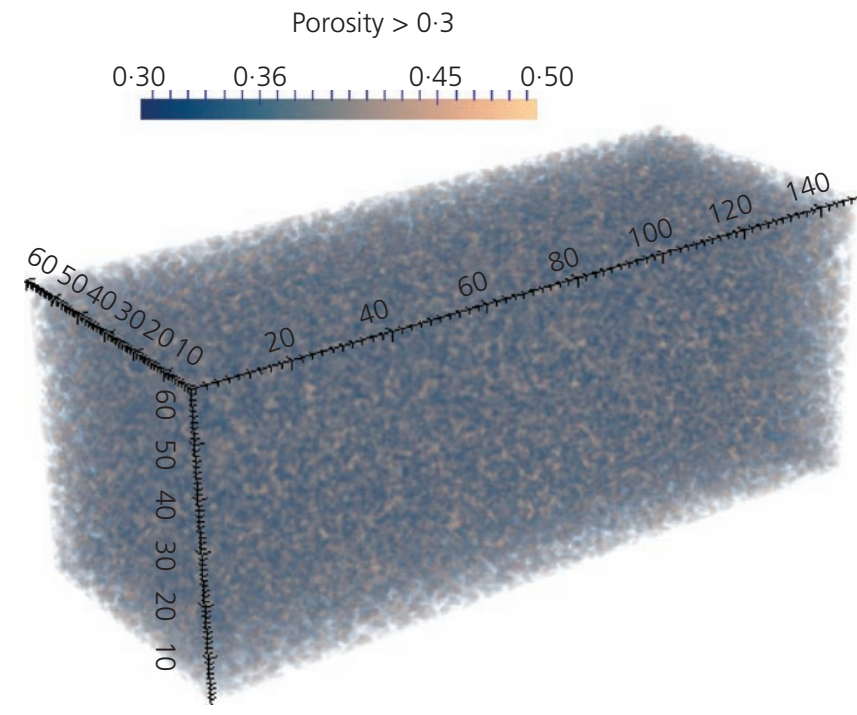

(a)

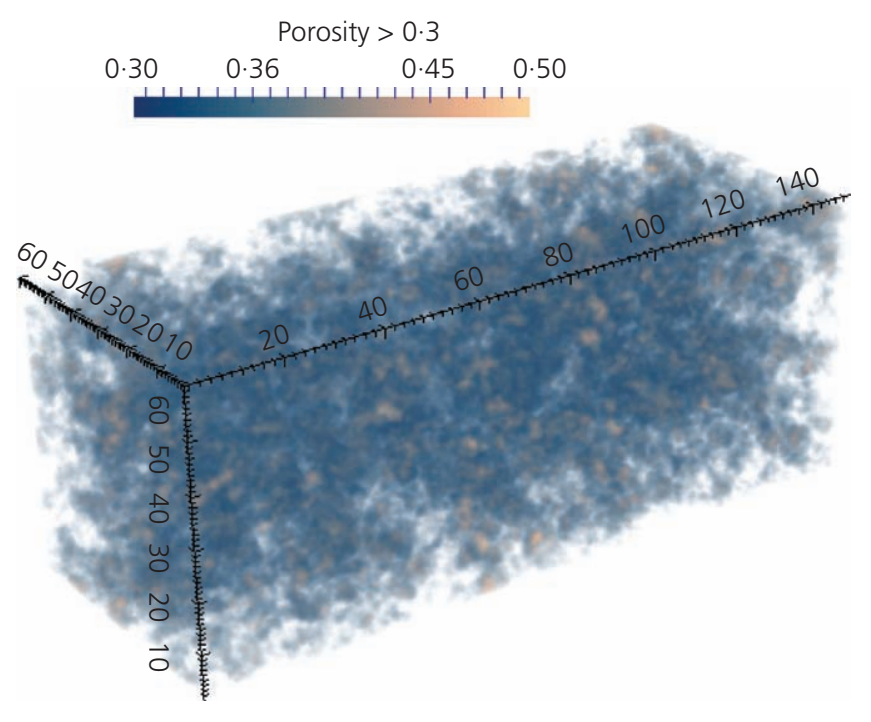

(b)

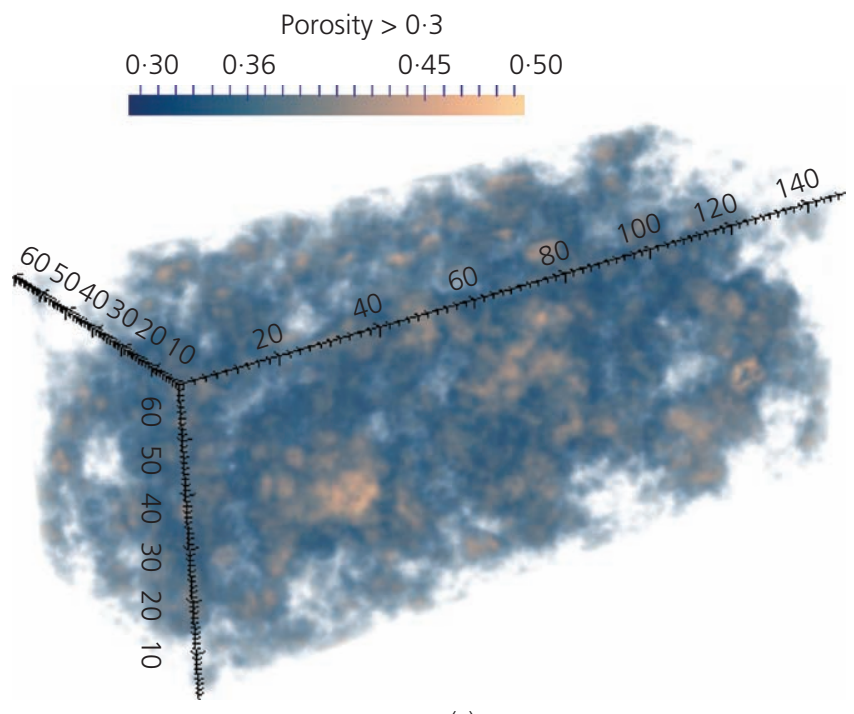

(c)

Figure 2. Three initial porosity fields $\left(\phi_{0}\right)$ that are used for wormholing calculation in this study: (a) randomly generated field; (b) spatially correlated field with $\lambda_{x, y, z}=0.0667 \mathrm{~cm}$; (c) spatially correlated field with $\lambda_{x, y, z}=0.1667 \mathrm{~cm}$ 
Environmental Geotechnics

Volume 8 Issue 1
The effects of spatially correlated

heterogeneities on acidising wormhole

development

Babaei and Sedighi
The correlated porosity fields are generated using $\mu_{\phi_{0}}=0 \cdot 27$ and $\sigma_{\phi_{0}}^{2}$ of $0 \cdot 0045$, so that the mean of porosity is comparable with those of existing simulation studies (Panga et al. (2005), Ghommem et al. (2015) and Liu et al. (2016): $\left.\mu_{\phi_{0}}=0 \cdot 20\right)$. Two correlation lengths $\left(\lambda_{x, y, z}\right)$ of two and five gridblocks (i.e. $0 \cdot 0667$ and $0.1667 \mathrm{~cm}$ ) are used to generate the correlated fields The correlation lengths in each direction are equal - that is, $\lambda_{x}=\lambda_{y}=\lambda_{z}-$ so that the field is isotropically correlated. To make a comparison, the authors also generate a random distribution of porosity with $\mu_{\phi_{0}}$ of 0.27 and $\sigma_{\phi_{0}}^{2}$ of 0.0045 . Therefore, in total, three porosity fields are considered for comparison as shown in Figure 2. The authors note that, in this figure, only porosities larger than 0.3 are shown to make the correlated features inside the domain visible. Also, the authors note that the axes' labels refer to the gridblock numbers and not the physical lengths scales.

The histograms of the porosity fields under comparison are shown in Figure 3. There is a clear difference between the distribution of the uncorrelated random field and correlated fields. The correlated fields demonstrate a realistic representation of porosity (e.g.
Figure 5 of the paper by Sahin et al. (2003)) for the porosity distribution for an Upper Jurassic carbonate reservoir located in the Eastern Province of Saudi Arabia). As such, the uniform distribution of a random porosity field - which is commonly used in the literature of acidisation simulation - is not realistic. The three initial porosity fields make the pore volume (PV) of three simulations equal to $5.003,5.5479$ and $5.8085 \mathrm{~cm}^{3}$. The difference between these values will not make the authors' comparisons inconsistent, because they use pore volume injected to create a non-dimensional injection rate.

Using the porosity fields, the authors generate initial permeability fields $\left(K_{0}\right)$ using a simple scaling so that the permeabilities are between 0 and $100 \mathrm{mD}\left(9 \cdot 869233 \times 10^{-14} \mathrm{~m}^{2}\right)$. A constant inlet velocity of $u_{0}$ is used which is equal to a constant flow rate of $1 \mathrm{~cm}^{3} / \mathrm{s}$ divided by the cross-sectional area of the core sample, which is $4 \mathrm{~cm}^{2}$. Therefore, $u_{0}$ is $0.25 \mathrm{~cm} / \mathrm{s}$. Using an effective molecular diffusivity of acid equal to $3 \times 10^{-9} \mathrm{~m}^{2} / \mathrm{s}$ (Liu et al., 2016), the macroscopic Péclet number is $4.17 \times 10^{4}$ in the authors' simulations. The large Péclet number lies on the upper limits of experiments carried out by Panga et al. (2005); therefore,

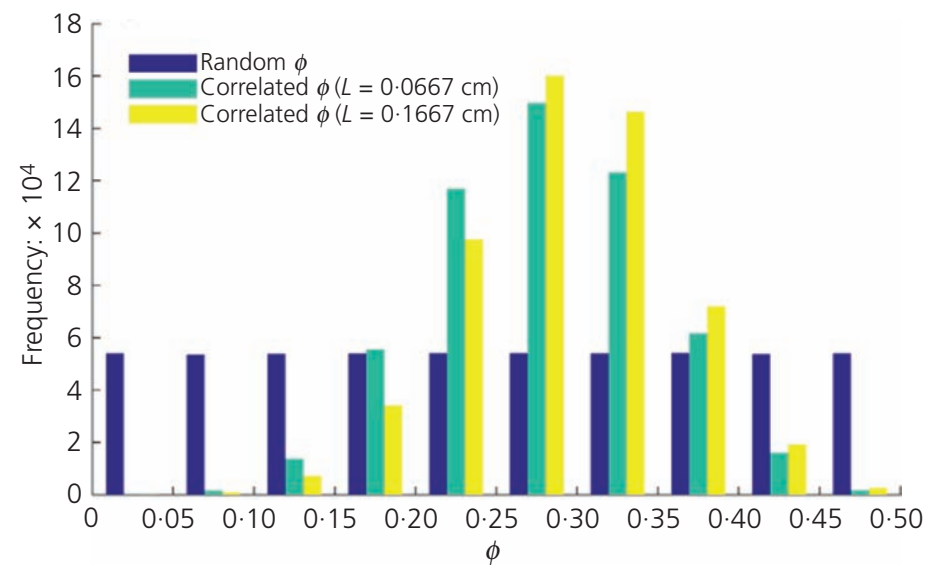

(a)

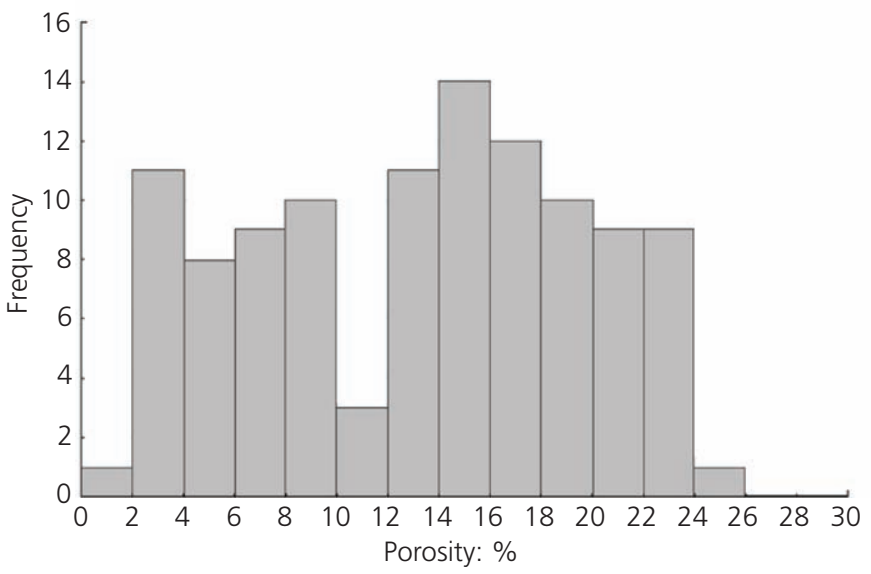

(b)

Figure 3. (a) The histogram of three porosity fields under study; (b) a realistic sample case adopted from the paper of Sahin et al. (2003) 
the generation of wormholes will depend only on the magnitude of the Damköhler number.

The finite-difference and implicit pressure and explicit saturation schemes are employed to solve the pressure and continuity equations (Equations 4 and 5) and the concentration equation (Equation 6) (Babaei and Sedighi, 2018). A $150 \times 60 \times 60$ domain (gridblock dimensions are $0.0333^{3} \mathrm{~cm}^{3}$ ) is used to discretise the governing equations. The criterion for the conditions in which wormholes can be considered to have been developed fully is not universally defined in different literature studies. While many authors use a greater-than-a-threshold breakthrough concentration of acid as an indication of wormhole development, one can argue that this may happen in non-wormholing dissolution regimes as well. To this end, the authors use the pressure gradient across the domain in the flow direction as a practical and reliable criterion. More specifically, they use the ratio of pressure difference at any simulation time $t$ over the initial pressure gradient $\left(\Delta p_{t} / \Delta p_{0}\right)$ as a measure of how effectively wormholes could reduce the pressure difference. If the dimensionless pressure drop $\left(\Delta p_{t} / \Delta p_{0}\right)$ has become less than or equal to $10^{-4}$, the authors assume the wormholes are generated and have fully grown to create superconductive flow paths so that the pressure difference has dropped to almost zero across the domain. The simulations are continued as long as either the dimensionless pressure drop reaches the threshold of $1 \times 10^{-4}$ or the pore volume of injected acid (PVI) reaches 4 . Both these values are chosen arbitrarily/heuristically, but as will be shown in the next section, these values lead to effective identification of wormhole generation.

\section{Results and discussions}

The authors base their comparisons on nine simulation scenarios: three porosity-permeability fields and three Damköhler regimes of $\mathrm{Da}=10, \mathrm{Da}=100$ and $\mathrm{Da}=1000$. In all nine simulation cases, thanks to a high Péclet number, wormholes are generated (no face or uniform dissolution patterns are observed). Figure 4 shows how wormholes are generated for the simulation scenarios. There is clearly a pattern shift from a low Da of 10 to a Da of 1000 . The wormholes are ramified for a low $\mathrm{Da}$, and the porosity increase within the wormholes is around 0.5 . Increasing $\mathrm{Da}$ to 100 , the wormholes are sharpened (so that a reduced amount of acid is consumed), yet still ramified. Finally, for $\mathrm{Da}=1000$, the wormholes are sharp and effective, consuming the least amount of acid. In terms of comparing the wormholes between the different porosity fields, the authors have to first note that Figures 4(a)-4(c) are produced at PVI $=4$ (because $\Delta p_{t} / \Delta p_{0}$ does not reach $10^{-4}$ ), whereas Figures 4(d)-4(i) are produced exactly when the dimensionless pressure drop has reached $10^{-4}$. Therefore, the profiles shown in Figures 4(d)-4(i) are not generated for the same

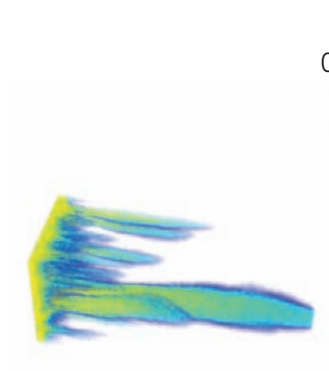

(a)

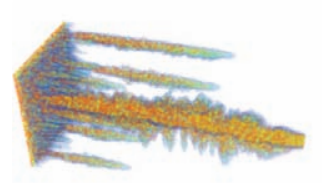

(d)

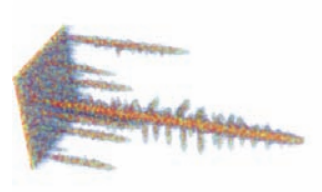

(g)

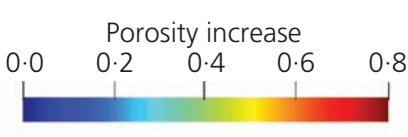

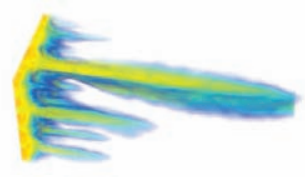

(b)

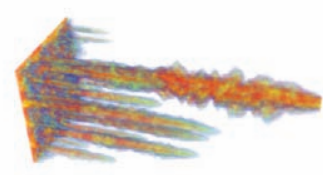

(e)

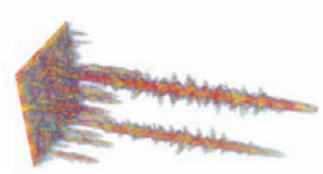

(h)

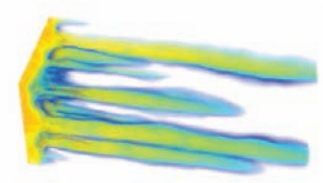

(c)

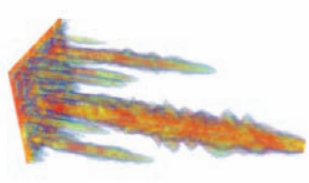

$(f)$

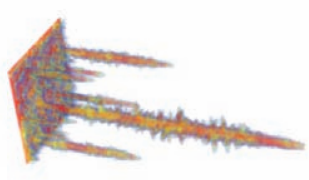

(i)

Figure 4. Absolute porosity increase when (a-c) PVI reaches 4 or $(d-i) \Delta p_{t} / \Delta p_{0}$ reaches $1 \times 10^{-4}$ for the random porosity (the left column), the correlated porosity with $\lambda_{x, y, z}=0.0667 \mathrm{~cm}$ (the middle column) and the correlated porosity with $\lambda_{x, y, z}=0 \cdot 1667 \mathrm{~cm}$ (the right column): $(\mathrm{a}-\mathrm{c}) \mathrm{Da}=10$; (d-f) Da = 100; (g-i) Da $=1000$ 


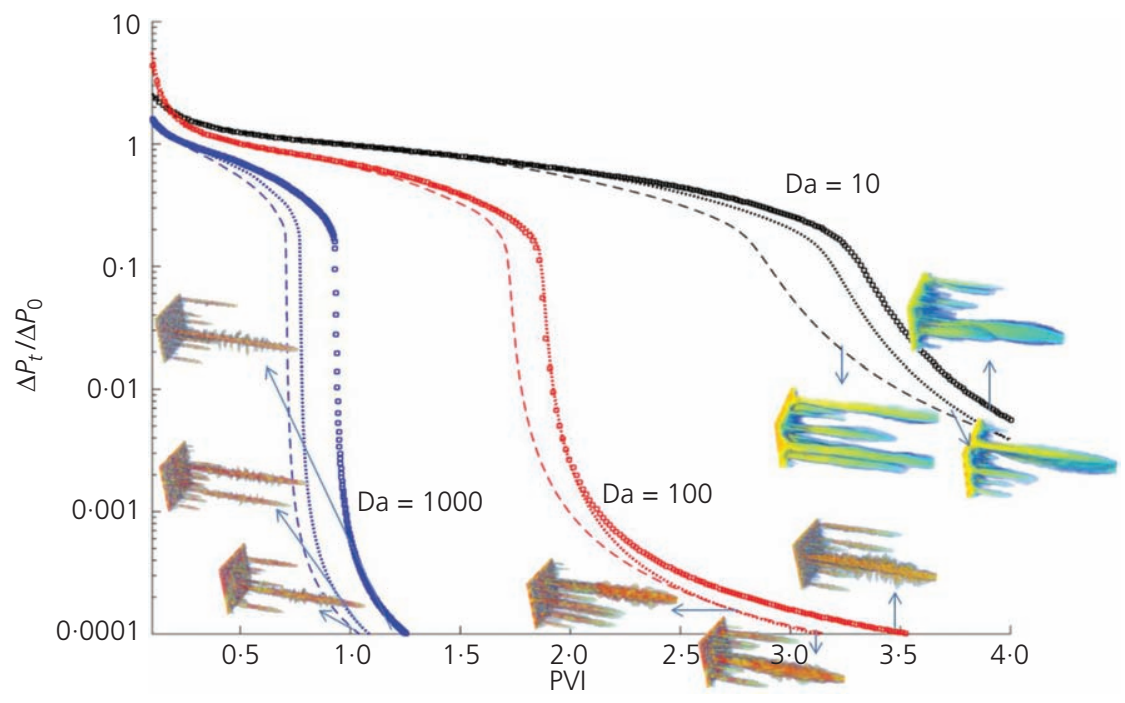

Figure 5. Profiles of the dimensionless pressure drop $\left(\Delta p_{t} / \Delta p_{0}\right)$ for nine simulation scenarios. The circles, the dotted lines and the dashed lines represent the profiles of the random porosity, the correlated porosity with $\lambda_{x, y, z}=0.0667 \mathrm{~cm}$ and the correlated porosity with $\lambda_{x, y, z}=$ $0.1667 \mathrm{~cm}$, respectively. Da regimes are grouped together with labels $\mathrm{Da}=1,100$ and 1000

amount of acid injected and show only how the wormholes have developed when the simulations are actually terminated.

In Figure 5, the amount of acid consumed in terms of PVI is plotted for different simulation scenarios. The figure clearly shows that moving towards the correlated porosity fields, the PVI required to hit the threshold of $\Delta p_{t} / \Delta p_{0}=10^{-4}$ is decreased for $\mathrm{Da}=100$ and $\mathrm{Da}=1000$. In Table 3, decreases in PVI for $\mathrm{Da}=$ 100 and $\mathrm{Da}=1000$ are recorded. One can observe that the correlated field with $\lambda_{x, y, z}=0 \cdot 1667 \mathrm{~cm}$ has decreased the PVI by $12 \cdot 2 \%$ for $\mathrm{Da}=100$ and by $17 \cdot 6 \%$ for $\mathrm{Da}=1000$ relative to the random field. These relative reductions are more considerable compared to the relative reductions in PVI when the randomly generated heterogeneity $\left(\Delta \phi_{0} / \Delta \phi_{0}\right)$ is increased from 0.5 to 1.5 (see Figure 6 of the paper by Panga et al. (2005)). There, the authors showed that the increased heterogeneity will have a maximum $10 \%$ reduction in the PVI for a wormhole regime of $\mathrm{Da}=1000$ (see Figure 7 of the paper by Panga et al. (2005)).

The reason why the correlated fields facilitate a faster and more effective growth of wormholes is that the correlated porosity-permeability allows either fewer branches to develop from

Table 3. PVI to make $\Delta p_{t} / \Delta p_{0}=10^{-4}$ for $\mathrm{Da}=100$ and $\mathrm{Da}=1000$ and the relative decrease with respect to the random porosity field

\begin{tabular}{lcc} 
& $\mathrm{Da}=\mathbf{1 0 0}$ & $\mathrm{Da}=\mathbf{1 0 0 0}$ \\
\hline $\begin{array}{l}\text { Random porosity } \\
\text { Correlated porosity }(0.0667 \mathrm{~cm}) \\
\text { (\% of PVBT decrease with respect }\end{array}$ & $3.15(10.5 \%)$ & $1.08(13.6 \%)$ \\
$\quad$ to the random porosity field) & & \\
$\begin{array}{l}\text { Correlated porosity }(0.1667 \mathrm{~cm})(\% \\
\text { of PVBT decrease with respect to } \\
\text { the random porosity field) }\end{array}$ & $3.09(12.2 \%)$ & $1.03(17.6 \%)$ \\
\hline
\end{tabular}

the wormholes or fewer unsuccessful wormholes to generate. The spatial correlation in porosity and permeability favours acid front movement longitudinally and suppresses to some extent its lateral movement (branching). This is more obvious when comparing the porosity increase snapshots of Figure 4(d) with Figure 4(f) for $\mathrm{Da}=100$ or Figure 4(g) with Figure 4(i) for $\mathrm{Da}=1000$.

To inspect the effect of correlation length on wormhole geometry (branchiness, thickness and straightness), in Figure 6, the ratio of permeability $(\kappa)$ is compared between the random porosity field and the correlated porosity with $\lambda_{x, y, z}=0 \cdot 1667 \mathrm{~cm}$, for $\mathrm{Da}=100$ and $\mathrm{Da}=1000$ when $\Delta p_{t} / \Delta p_{0}=10^{-4}$. Similar to the porosity increase, the snapshots show that the following.

- The sizes of branches for the random porosity field are slightly larger than the correlated porosity field for both $\mathrm{Da}=$ 100 and $\mathrm{Da}=1000$.

- The number of undeveloped wormholes for the random porosity field are slightly larger than the correlated porosity field for both $\mathrm{Da}=100$ and $\mathrm{Da}=1000$.

Therefore, both variables - that is, the porosity increase and the ratio of dimensionless permeability over initial permeability indicate that for a small system of $5 \mathrm{~cm}$, the effects of correlation length on petrophysical properties are tangible. It should be noted that the effect of correlation in the porosity/permeability and the relative reduction in $\mathrm{PV}_{\mathrm{BT}}$ (or PVI to make $\Delta p_{t} / \Delta p_{0}=10^{-4}$ ) should be higher for larger model samples - for example, when $L=50 \mathrm{~cm}$.

\section{Conclusions and future works}

The findings of this work have clear practical relevance to control and design of acidising operations where the aim is to enhance hydrocarbon or heat recovery from hydrocarbon or geothermal 


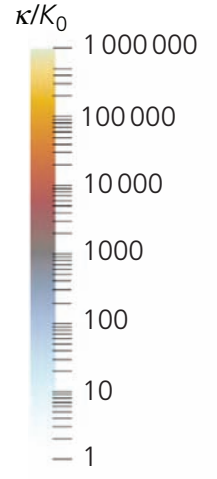

(a)

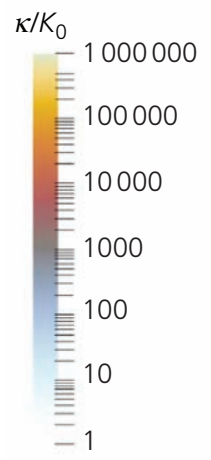

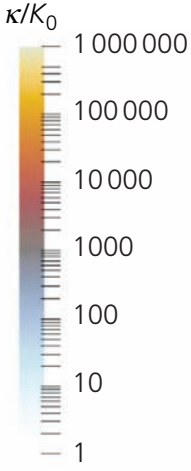

(b)

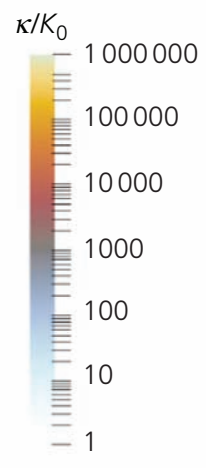

Figure 6. The dimensionless permeability ratio for (a) the random porosity field, $\mathrm{Da}=100$ at $\mathrm{PVI}=3 \cdot 52$; (b) the porosity field with $\lambda_{x, y z}=$ $0 \cdot 1667 \mathrm{~cm}, \mathrm{Da}=100$ at $\mathrm{PVI}=3 \cdot 09$; (c) the random porosity field, $\mathrm{Da}=1000$ at $\mathrm{PVI}=1 \cdot 25$; and (d) the porosity field with $\lambda_{x, y, z}=0 \cdot 1667 \mathrm{~cm}$, $\mathrm{Da}=100$ at $\mathrm{PVI}=1.03$ 
reservoirs. An inevitable existence of correlated features in porous media of these systems means that an acid operation can be carried out more efficiently by consuming less acid. For example, for a target area around the wellbore, $17 \cdot 6 \%$ less PVI translates into several cubic metres less acid consumed. Moreover, ignoring the spatial correlation may result in overconsuming and jeopardising the integrity of reservoir barriers or cap rock, consequently leading to potential contamination of underground water resources by acidic solutions. So far, the objective of most of the acid wormholing modelling works has matched the core sample experiments. However, the spatial correlation of porous media petrophysical properties - that span beyond the core and mesoscopic scale into reservoir scale - should be incorporated in modelling-based operation design.

The acid treatment studied in this work did not include the effects of secondary phases/fluids remaining from a pre-flush stage. However, recent publications, including that of Babaei and Sedighi (2018), have investigated the presence of such phases (e.g. oil blobs or trapped air) on the wormholing process. The investigation (on uniformly distributed porosity fields) shows that the secondary phase will lead to less branchy wormholes and as a result will enhance wormhole development - the results that are supported by experiments (e.g. Shukla et al., 2006). An investigation to depict the interplay of realistic heterogeneity of porous rock (with spatial correlation) and two-phase flow conditions is a of matter of interest in future studies.

Finally, the mechanical stability of developed wormholes and their dependence on the correlation lengths of the porous system are very important in the design of the acidising operation. The process of effective wormhole generation and its stability during the production period are a coupled phenomenon that requires studying the reactive transport and mechanics of the problem alongside each other. During the generation of wormholes, pore fluid pressure withstands the overburden pressure. However, excessive lateral growth of the wormholes and loss of acid solution to rock could generate geomechanical instability. Under this condition, the wormholes become susceptible to collapse. In this line of research, optimising acid reaction rates, amount, acidity and level of interaction with rock are key factors in obtaining the desired effects on the formation at downhole conditions. Coupling of thermohydromechanical effects (e.g. Salimzadeh et al., 2018) is required to determine optimal condition for sustainable acidisation.

\section{Acknowledgements}

The development of the $3 \mathrm{D}$ continuum scale code for reactive transport processes was achieved by the support of Engineering and Physical Sciences Research Council First Grant EP/R009678/1 that was awarded to MB.

\section{REFERENCES}

Alkattan M, Oelkers EH, Dandurand JL and Schott J (1998) An experimental study of calcite and limestone dissolution rates as a function of $\mathrm{pH}$ from -1 to 3 and temperature from 25 to $80^{\circ} \mathrm{C}$. Chemical Geology 151(1-4): 199-214, https://doi.org/10.1016/S00092541(98)00080-1.

Atchley AL, Navarre-Sitchler AK and Maxwell RM (2014) The effects of physical and geochemical heterogeneities on hydro-geochemical transport and effective reaction rates. Journal of Contaminant Hydrology 165: 53-64.

Babaei M and Sedighi M (2018) Impact of phase saturation on wormhole formation in rock matrix acidizing. Chemical Engineering Science 177: 39-52.

Bastami A and Pourafshary P (2016) Development of a new model for carbonate matrix acidizing to consider the effects of spent acid. Journal of Energy Resources Technology 138(5): 052905.

Chen JS, Liu CW, Lai GX and Ni CF (2009) Effects of mechanical dispersion on the morphological evolution of a chemical dissolution front in a fluid-saturated porous medium. Journal of Hydrology 373(1): 96-102.

Cohen CE, Ding DY, Quintard M and Bazin B (2007) A new matrix acidizing simulator based on a large scale dual porosity approach. Proceedings of the European Formation Damage Conference. The Hague, the Netherlands, paper no. SPE-107755-MS.

Cohen CE, Ding D, Quintard M and Bazin B (2008) From pore scale to wellbore scale: impact of geometry on wormhole growth in carbonate acidization. Chemical Engineering Science 63(12): 3088-3099.

Cubillas P, Köhler S, Prieto M, Causserand C and Oelkers EH (2005) How do mineral coatings affect dissolution rates? An experimental study of coupled $\mathrm{CaCO}_{3}$ dissolution- $\mathrm{CaCO}_{3}$ precipitation. Geochimica et Cosmochimica Acta 69(23): 5459-5476.

Daccord G, Touboul E and Lenormand R (1989) Carbonate acidizing: toward a quantitative model of the wormholing phenomenon. Society of Petroleum Engineers 4(1): 63-68.

De Oliveira TJL, de Melo AR, Oliveira JAA and Pereira AZ (2012) Numerical simulation of the acidizing process and PVBT extraction methodology including porosity/permeability and mineralogy heterogeneity. Proceedings of the SPE International Symposium and Exhibition on Formation Damage Control, Lafayette, LA, USA, paper no. SPE-151823-MS.

Evans RL and Lizarralde D (2003) Geophysical evidence for karst formation associated with offshore groundwater transport: an example from North Carolina. Geochemistry, Geophysics, Geosystems 4(8).

Fredd CN and Fogler HS (1998) Influence of transport and reaction on wormhole formation in porous media. AIChE Journal 44(9): 1933-1949.

Ghommem M, Zhao W, Dyer S, Qiu X and Brady D (2015) Carbonate acidizing: modeling, analysis, and characterization of wormhole formation and propagation. Journal of Petroleum Science and Engineering 131: 18-33.

Glasbergen G, Kalia N and Talbot MS (2009) The optimum injection rate for wormhole propagation: myth or reality? Proceedings of the 8th European Formation Damage Conference, The Hague, the Netherlands, paper no. SPE-121464-MS.

Golfier F, Quintard M, Bazin B and Lenormand R (2006) Core-scale description of porous media dissolution during acid injection-part II: calculation of the effective properties. Computational \& Applied Mathematics 25(1): 55-78.

Hung KM, Hill AD and Sepehrnoori K (1989) A mechanistic model of wormhole growth in carbonate matrix acidizing and acid fracturing. Journal of Petroleum Technology 41(1): 59-66.

Islam A, Kazemi Nia Korrani A, Sepehrnoori K and Patzek T (2014) Effects of geochemical reaction on double diffusive natural convection of $\mathrm{CO}_{2}$ in brine saturated geothermal reservoir. Energy Procedia 63: 5357-5377.

Islam A, Sun A and Lu J (2016) Simulating in-zone chemistry changes from injection time to longer periods of $\mathrm{CO}_{2}$ storage. Environmental Earth Sciences 75(20): 1346, https://doi.org/10.1007/s12665-016-6153-9. 
Izgec O, Keys RS, Zhu D and Hill AD (2008) An integrated theoretical and experimental study on the effects of multiscale heterogeneities in matrix acidizing of carbonates. Proceedings of the SPE Annual Technical Conference and Exhibition, Denver, CO, USA, paper no. SPE-115143-MS.

Izgec O, Zhu D and Hill AD (2010) Numerical and experimental investigation of acid wormholing during acidization of vuggy carbonate rocks. Journal of Petroleum Science and Engineering 74(1-2): 51-66, https://doi.org/10.1016/j.petrol.2010.08.006.

Kalia N and Balakotaiah V (2007) Modeling and analysis of wormhole formation in reactive dissolution of carbonate rocks. Chemical Engineering Science 62(4): 919-928.

Kalia N and Balakotaiah V (2009) Effect of medium heterogeneities on reactive dissolution of carbonates. Chemical Engineering Science 64(2): 376-390.

Kampman N, Bickle M, Wigley M and Dubacq B (2014) Fluid flow and $\mathrm{CO}_{2}$-fluid-mineral interactions during $\mathrm{CO}_{2}$-storage in sedimentary basins. Chemical Geology 369: 22-50.

Liu N and Liu M (2016) Simulation and analysis of wormhole propagation by VES acid in carbonate acidizing. Journal of Petroleum Science and Engineering 138: 57-65.

Liu M, Zhang S and Mou J (2012) Effect of normally distributed porosities on dissolution pattern in carbonate acidizing. Journal of Petroleum Science and Engineering 94: 28-39.

Liu PL, Xue H, Zhao LQ, Zhao XD and Cui MY (2016) Simulation of 3D multi-scale wormhole propagation in carbonates considering correlation spatial distribution of petrophysical properties. Journal of Natural Gas Science and Engineering 32: 81-94.

Liu P, Yao J, Couples GD et al. (2017) Modelling and simulation of wormhole formation during acidization of fractured carbonate rocks. Journal of Petroleum Science and Engineering 154: 284-301, https://doi.org/10.1016/j.petrol.2017.04.040.

Maheshwari P and Balakotaiah V (2013a) 3D simulation of carbonate acidization with $\mathrm{HCl}$ : comparison with experiments. Proceedings of the SPE Production and Operations Symposium, Oklahoma City, OK, USA, paper no. SPE-164517-MS.

Maheshwari P and Balakotaiah V (2013b) Comparison of carbonate $\mathrm{HCl}$ acidizing experiments with 3D simulations. SPE Production \& Operations 28(4): 402-413.

Maheshwari P, Ratnakar RR, Kalia N and Balakotaiah V (2013) 3-D simulation and analysis of reactive dissolution and wormhole formation in carbonate rocks. Chemical Engineering Science $\mathbf{9 0}$ $258-274$

Na J, Xu T, Jiang Z et al. (2016) A study on the interaction of mud acid with rock for chemical stimulation in an enhanced geothermal system. Environmental Earth Sciences 75(12): 1-13.

Nierode DE, Williams BR and Bombardieri CC (1972) Prediction of stimulation from acid fracturing treatments. Journal of Canadian Petroleum Technology 11(4).

Nowak W, Schwede RL, Cirpka OA and Neuweiler I (2008) Probability density functions of hydraulic head and velocity in three-dimensional heterogeneous porous media. Water Resources Research 44(8).

Ouhadi VR, Yong RN and Sedighi M (2006) Influence of heavy metal contaminants at variable $\mathrm{pH}$ regimes on rheological behavior of bentonite. Applied Clay Science 32: 217-231.

Panga MKR, Ziauddin M and Balakotaiah V (2005) Two-scale continuum model for simulation of wormholes in carbonate acidization. AIChE Journal 51(12): 3231-3248.

Portier S and Vuataz FD (2010) Developing the ability to model acid-rock interactions and mineral dissolution during the RMA stimulation test performed at the Soultz-sous-Forêts EGS site, France. Comptes Rendus Geoscience 342(7): 668-675.

Portier S, André L and Vuataz FD (2007) Review on Chemical Stimulation Techniques in Oil Industry and Applications to Geothermal Systems. Center for Geothermal Research, Neuchâtel, Switzerland.
Qiu X, Zhao W, Chang F and Dyer S (2013) Quantitative modeling of acid wormholing in carbonates - what are the gaps to bridge. Proceedings of the SPE Middle East Oil and Gas Show and Conference, Manama, Bahrain, paper no. SPE-164245-MS

Rochelle CA, Czernichowski-Lauriol I and Milodowski AE (2004) The impact of chemical reactions on $\mathrm{CO}_{2}$ storage in geological formations: a brief review. Geological Society, London, Special Publications 233(1): 87-106

Sahin A, Menouar H, Ali AZ and Saner S (2003) Patterns of variation of permeability anisotropy in a carbonate reservoir. Proceedings of the 13th SPE Middle East Oil Show and Conference, Bahrain, paper no. SPE-81472-MS.

Salimzadeh S, Paluszny A, Nick HM and Zimmermann RW (2018) A threedimensional coupled thermo-hydro-mechanical model for deformable fractured geothermal systems. Geothermics 71: 212-224.

Schechter RS and Gidley JL (1969) The change in pore size distribution from surface reactions in porous media. AIChE Journal 15(3): 339-350.

Sedighi M, Thomas HR, Al Masum S et al. (2015) Geochemical modelling of hydrogen gas migration in an unsaturated bentonite buffer. Geological Society, London, Special Publications 415(1): 189-201.

Sedighi M, Thomas HR and Vardon PJ (2018) Reactive transport of chemicals in compacted bentonite under non-isothermal water infiltration. Journal of Geotechnical and Geoenvironmental Engineering 144(10): p. 04018075, https://doi.org/10.1061/(ASCE)GT. 1943-5606.0001955.

Shukla S, Zhu D and Hill AD (2006) The effect of phase saturation conditions on wormhole propagation in carbonate acidizing. SPE Journal 11(3): 273-281.

Spycher NF, Sonnenthal EL and Apps JA (2003) Fluid flow and reactive transport around potential nuclear waste emplacement tunnels at Yucca Mountain, Nevada. Journal of Contaminant Hydrology 62: 653-673.

Valdes-Abellan J, Jiménez-Martinez J, Candela L et al. (2017) Reactive transport modelling to infer changes in soil hydraulic properties induced by non-conventional water irrigation. Journal of Hydrology 549: 114-124.

Wyllie MRJ and Gardner GHF (1958) The generalized Kozeny-Carman equation. World Oil 146(4): 121-128.

Xiong Y, Fakcharoenphol P, Winterfeld P, Zhang R and Wu YS (2013) Coupled geomechanical and reactive geochemical model for fluid and heat flow: application for enhanced geothermal reservoir. Proceedings of the SPE Reservoir Characterization and Simulation Conference and Exhibition, Abu Dhabi, UAE, vol. 1, paper no. SPE-165982-MS.

Zhao C, Hobbs B and Ord A (2013) Theoretical analyses of acidization dissolution front instability in fluid-saturated carbonate rocks. International Journal for Numerical and Analytical Methods in Geomechanics 37(13): 2084-2105.

\section{How can you contribute?}

To discuss this paper, please submit up to 500 words to the editor at journals@ice.org.uk. Your contribution will be forwarded to the author(s) for a reply and, if considered appropriate by the editorial board, it will be published as a discussion in a future issue of the journal. 